\title{
Ubiquitous Assessment of the Recovery of Cancer Patients Using Consumer-Level Activity Trackers ${ }^{\dagger}$
}

\author{
Salvador Moreno ${ }^{1, *(1)}$, Miguel Damas ${ }^{1}$ (i), Ignacio Rojas ${ }^{1}$, Victor Amezcua ${ }^{2}$, \\ Pilar Gutierrez-Pastor ${ }^{2}$ and Oresti Banos ${ }^{1}$ (iD \\ 1 Department of Computer Architecture and Technology, CITIC-UGR Research Center, University of Granada, \\ 18071 Granada, Spain; mdamas@ugr.es (M.D.); irojas@ugr.es (I.R.); oresti@ugr.es (O.B.) \\ 2 Medical Oncology Service, Virgen de las Nieves Hospital, 18014 Granada, Spain; \\ euroncame@gmail.com (V.A.); pilargutipastor@gmail.com (P.G.-P.) \\ * Correspondence: salvadormoreno@ugr.es \\ $\dagger$ Presented at the 12th International Conference on Ubiquitous Computing and Ambient Intelligence \\ (UCAmI 2018), Punta Cana, Dominican Republic, 4-7 December 2018.
}

Published: 18 October 2018

\begin{abstract}
Performance Status (PS) variability is a powerful tool to evaluate overall condition, treatment needs and survival chances of cancer patients. Traditionally, its assessment has relied on the experience of oncologists when interpreting results of clinical tests and when interviewing the patients. Meanwhile, consumer-level activity trackers have obtained good results in behavior-change oriented intervention trials and Fitbit devices have demonstrated enough reliability to provide objective data related to physical activity, but the clinical possibilities of the data collected has been neglected. This work presents a system design for ubiquitous assessment of PS by means of objective and quantifiable data from different sources: medical history, self-reported quality-of-life questionnaires and a commercial activity tracker Fitbit Alta HR. The system proposed aims to contextualize and model the recovery process of breast cancer patients during chemotherapy treatment.
\end{abstract}

Keywords: ubiquitous; e-Health; chemotherapy; cancer; performance status; physical activity; Fitbit

\section{Introduction}

Performance Status (PS) variability is a powerful tool to evaluate overall condition, treatment needs and survival chances of cancer patients. It measures the ability of patients to perform the activities of daily living by comprehending several dimensions of the disease, such as physical activity performance, quality of life and the toxicity levels of the treatment [1]. The Eastern Cooperative Oncology Group (ECOG) [2] and Karnofsky Performance Status (KPS) [3] scales have served this purpose for decades and they are still widely used among oncologists.

However, the ambiguity and broad dimensions rooted in the definition of PS scales make them prone to subjectivity and interpretation, forcing oncologists to evaluate PS mainly from personal interviews with patients that may be under severe conditions of pressure, anxiety and uncertainty, along with the secondary effects of the treatment $[4,5]$. This subjectivity and the tight relationship of physical activity with PS [6] are the main reasons for introducing activitytrackers when monitoring the recovery of cancer patients.

Several clinical trials have already used wearable activity trackers in oncology trials, specially with breast cancer patients and survivors [7]. Research-grade monitors like Actigraph GT3X+ or the ActivPAL were the most used, but there is a growing trend in including consumer-level activity trackers like Fitbit devices due to their popularity, reliability and cost-effectiveness. Fitbit devices have been successfully used within the context of behavior change interventions such as healthy lifestyle promotion, physical activity raise and weight control in population with different conditions such 
as breast cancer survivors, pregnant moms and patients with type 2 diabetes mellitus [8-10], but the possible clinical relevance of the data collected is still being neglected. Despite the subtle differences interpreting results across the literature, most of them point out that Fitbit devices may provide reliable objective data referred to physical activity, especially within free living environments [11-14].

All this lays down the basis for the system proposed, where commercial activity trackers, namely Fitbit, provide ubiquitous, objective and quantifiable measures of the physical activity of breast cancer patients, thus aiming to reduce biases and subjectivity in PS evaluation.

\section{State of the Art}

In previous work we addressed automatic monitoring of PS from a broad perspective of cancer patients [15]. In this work we focus in breast cancer patients since they have been widely studied with research-oriented and commercial activity trackers. According to Gresham et al., they represented the $65 \%$ of the total amount of oncology trials related to activity trackers between 2005 and 2016 [7]. This over-representation of breast cancer (both patients and survivors) across oncology trials matches their high involvement and implication in their treatment and recovery. Moreover, the overall good acceptance reported ( $82 \%$ median adherence) is similar to the those reported in more recent studies promoting healthy lifestyle behavior in breast cancer patients (92.13\% adherence) [16] and survivors (88.13\% adherence) [8]. For these reasons, breast cancer patients reveal themselves as the most appropriate group to study the ubiquitous assessment of PS.

Regarding consumer-level activity trackers, Fitbit devices reported better reliability than the one we used in previous work [15,17], however, despite their promising results and growing acceptance in clinical trials, "discretion should be used when considering the use of Fitbit devices as an outcome measurement tool in research or to inform health care decisions" [17]. In this section we discuss some of the most relevant contributions related to the reliability provided by Fitbit devices: steps count, energy expenditure, physical activity levels, heart rate and sleep tracking.

\subsection{Fitbit's Steps Count}

Steps count is one of the main variables used for measuring physical activity levels, even in oncology trials $[7,18]$. The good accuracy reported by wrist-worn Fitbit devices in controlled and free-living settings makes this activity trackers ideal for the proposed system [17]. Some trials included in the systematic review of Feehan et al. [17] represent the conditions to be encountered when assessing PS in breast cancer patients, for example, a trial with 22 prostate cancer survivors monitored with Fitbit One during 7 days under free-living settings found no significant differences in steps count compared to GT3X+ $(r=0.94)$ [12]; and 22 healthy women monitored with Fitbit Flex during 7 days found no statistical difference in daily steps count compared GT3X+ [19].

On the other hand, the work of Ummels et al. rejected the validity of 9 commercial activity trackers (Fitbit One and Fitbit Flex among them) for 130 patients with a chronic disease [20]. However, the activities described in the protocol were most of them homework tasks, and very short in duration (19 activities in 28 to $33 \mathrm{~min}$ ). The short-lasting activities and the implicit low-consistent patterns of movement within them (e.g., $1 \mathrm{~min}$ of simulated cleaning of windows, $7 \mathrm{~m}$ of walking weaving around cones) may have over-hindered the steps count for the tested devices; besides, not including research-oriented activity trackers impedes any comparison to trials relying on validated monitors like GT3X+. Other works show that the validation problem of consumer-level devices does not seem to be rooted in the chronic condition of patient, but in their overall mobility [17], for example, Alharbi et al. obtained a very high correlation of Fitbit Flex steps count compared to GT3X+ $(r=0.95)$ in a community-based phase III cardiac rehabilitation population [21].

\subsection{Fitbit's Energy Expenditure \& Physical Activity Levels}

There is agreement on Fitbit overestimating energy expenditure and misclassifying physical activity levels (sedentary, light, moderate, vigorous) when compared to GT3X+, especially for moderate 
and vigorous activity $[12,13,17,19,22]$. Winfree et al. have successfully tackled this issue with a Naïve-Bayes classification model that leverages Fitbit Flex data as its input that removes statistical differences between GT3X+ MVPA (moderate-to-vigorous physical activity) and Fitbit Flex MVPA [23]. This approach makes shows that it is possible to extract meaningful knowledge from the original data provided by Fitbit, so this deserves to be studied along with reliable clinical data.

\subsection{Fitbit's Heart Rate $\mathcal{E}$ Sleep Tracking}

Regarding other measures of Fitbit such as heart rate, there is clear agreement in its low reliability and high sensitivity to artifacts and sensor displacement, so this data should be interpreted carefully $[11,17,24]$. Sleep tracking is also reported to be systematically overestimated [17], thus it needs to be addressed with caution too.

\section{System Design}

This section presents a system design based in previous work [15], adapting it to Fitbit devices and breast cancer patients. Figure 1 presents the system design for the experiment proposed. Patients will participate voluntarily through informed consent and always under the protection of General Data Protection Regulation (GDPR) guidelines; data will be anonymized and stored securely within a remote sever located in our faculty laboratory.

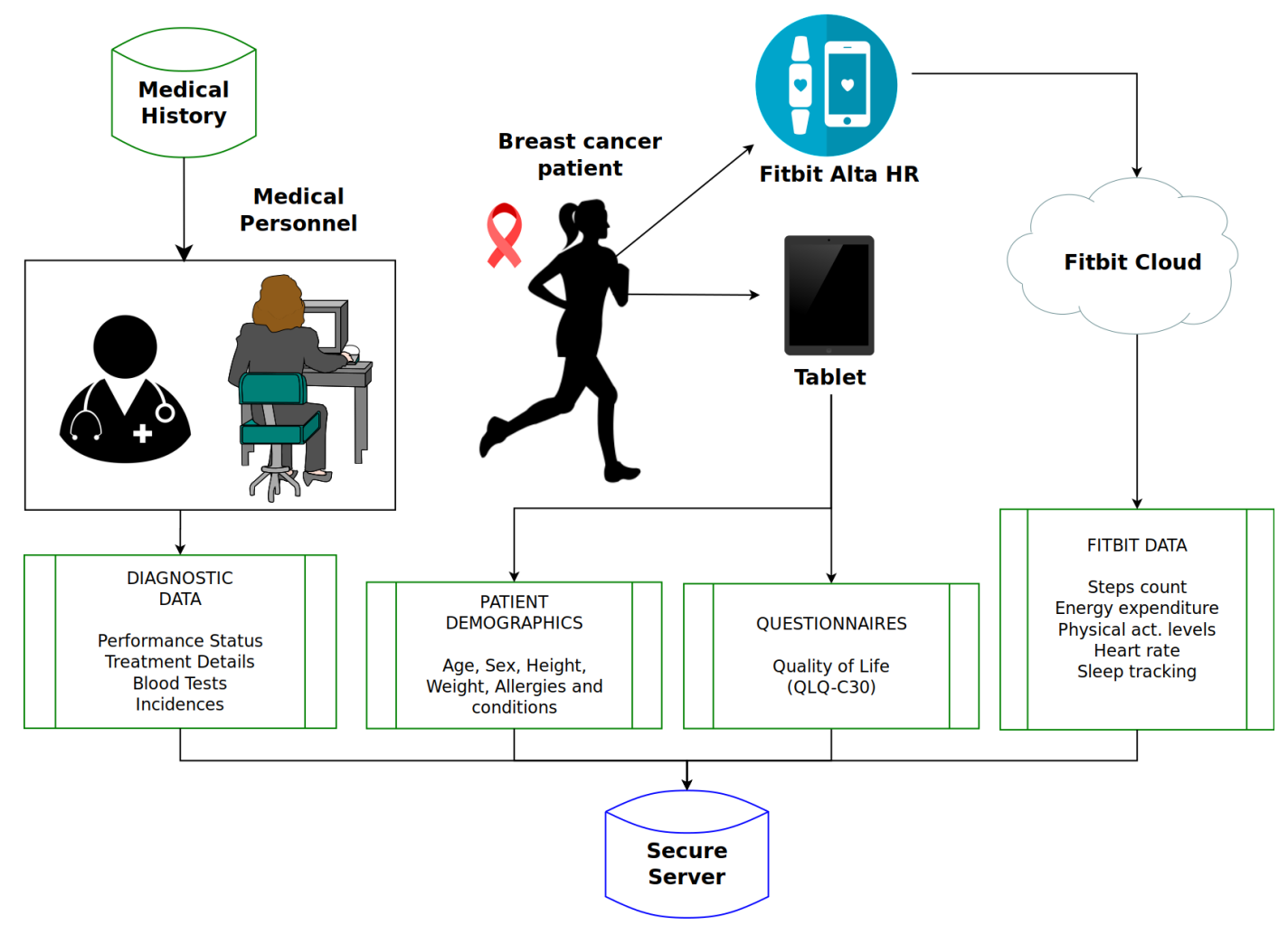

Figure 1. System design.

\subsection{Data Sources}

There are three main data sources: medical history, self-reported quality-of-life questionnaires and the Fitbit Cloud, which hosts the data collected by a Fitbit Alta HR activity tracker. On the left of Figure 1, there are data from medical records, the most important part of our system: the experts' knowledge. Clinical PS evaluations of patients will be the main outcome to measure; treatment details, blood tests and incidences will be gathered too during the recovery process to remove factors of confusion like anemia - which is related to sensations of fatigue and tiredness-- The frequency of 
these data will depend on the drugs and treatment administered, varying among 7, 14 or 21 days. This data needs introduced into our database manually through web forms connected to a secure web service.

Patient will report its demographics in the start of the trial when registering. They will report their quality of life with the QLQ-C30 questionnaire at the beggining, middle and end of their treatment to contrast self-reported functional and symptom scales. This data will be retrieved by using web forms connected to our database by a web service. The frequency of this data is even lower than medical records, but this methodology mimics previous intervention trials to avoid overwhelming patients with constant questionnaires.

Fitbit Alta HR will monitor the activity of each patient during the entire chemotherapy treatment (minimum of 12 weeks). This device measures every variable discussed in Section 2, so that will contextualize the data from medical history and questionnaires in an objective and quantifiable way. The sampling frequency of the device is very high (compared to the rest of the data) and it uploads all the data directly to the Fitbit Cloud. This cloud provides a REST API that allows data to be collected in our database with granularity from seconds (heart-rate) to minutes (steps count, energy expenditure, physical activity levels, sleep tracking).

\subsection{Data Security}

Security of patients is the most important issue while handling their sensitive data, therefore, this subsection explains the conditions in which data is stored and collected to meet security standards under GDPR legislation. To ensure physical security the server will be placed at the CITIC-UGR Research Center in the University of Granada (Granada, Spain). Access to the center will be restricted to university personnel, and besides, the server will be located in a room with limited access to the researchers involved in the project. The server will not be used by third persons in a direct nor indirect way. The information stored in the server will be encrypted so that there is no possible way to access the data, not even by stealing the hard drives or by other means. The database collecting the data will have limited access, only available to the research team. Any possible communication between a secure web environment (HTTPS) and the database will be made locally so that information is not visible nor accessible from the outside. Moreover, to ensure maximum security of external access, a firewall will limit the number of available ports for connections, only enabling ports 22 (SSH) and 443 (HTTPS). Both ports will be secured by OpenSSL libraries that ensure the confidentiality and integrity of the information by encrypting data exchanges with the outside.

\section{Discussion}

This work proposes a system to overcome the limitations of subjective PS evaluation by combining ubiquitous and objective physical activity data, clinically validated self-reported questionnaires (QLQ-C30) and the clinical data within medical records (e.g., PS assessed by oncologists, blood tests, symptoms, etc.). The consistency of Fitbit devices when measuring steps count, and the possibilities offered by energy expenditure and sleep tracking, support a more objective assessment of PS. Moreover, breast cancer patients reveal themselves as a trustworthy group to test the system proposed due to the high adherence and acceptance outcomes along activity-tracker related trials [7].

The inclusion of continuous and ubiquitous data of each patient will provide the ability to study it from a more individual perspective, focusing on tendencies and on the evolution of recovery across time. Diagnostic data will remain always as source of truth despite the critiques of PS scales, since the expert's knowledge is what we are aiming to support. The vision of this work is to ultimately provide oncologists with decision support systems for better assistance of cancer patients with affordable tools like commercial activity trackers. 


\section{Conclusions}

Performance status variability is a powerful tool to evaluate treatment needs and survival chances of cancer patients. Traditionally, its assessment has relied on personal interviews with patients and the experience of oncologists. Consumer-level activity trackers have managed good results among behavior-change oriented intervention trials, but the clinical possibilities of the data collected has been neglected. Fitbit devices have demonstrated enough reliability to supply objective data during the entire recovery process of breast cancer patients. This work presents a system design to evaluate performance status by means of objective and quantifiable data from different sources: medical history, self-reported quality-of-life questionnaires and continuous monitoring of a Fitbit Alta HR activity tracker. Its future implementation will allow us to extract the relevance of each variable in performance status assessment, and to model the recovery process of breast cancer patients during chemotherapy. A trial design with breast cancer patients and the system proposed has been approved by the Ethics Committee of the Virgen de las Nieves Hospital.

Funding: This work has been partially supported by the Spanish Ministry of Economy and Competitiveness (MINECO) Projects TIN2015-71873-R and TIN2015-67020-P together with the European Fund for Regional Development (FEDER). This work has also been partially supported by the FPU Spanish Grant FPU16/04201.

Conflicts of Interest: The authors declare no conflict of interest. The founding sponsors had no role in the design of the study; in the collection, analyses, or interpretation of data; in the writing of the manuscript, and in the decision to publish the results.

\section{References}

1. Yates, J.W.; Chalmer, B.; McKegney, F.P. Evaluation of patients with advanced cancer using the karnofsky performance status. Cancer 1980, 45, 2220-2224. doi:10.1002/1097-0142(19800415)45:8<2220:: AID-CNCR2820450835>3.0.CO;2-Q.

2. Zubrod, C.G.; Schneiderman, M.; Frei, E.; Brindley, C.; Lennard Gold, G.; Shnider, B.; Oviedo, R.; Gorman, J.; Jones, R.; Jonsson, U.; et al. Appraisal of methods for the study of chemotherapy of cancer in man: Comparative therapeutic trial of nitrogen mustard and triethylene thiophosphoramide. J. Chron. Dis. 1960, 11,7-33. doi:10.1016/0021-9681(60)90137-5.

3. Karnofsky, D.; Burchenal, J. The clinical evaluation of chemotherapeutic agents in cancer. In Evaluation of Chemotherapeutic Agents; Columbia Univ. Press: New York, NY, USA, 1949; pp. 191-205.

4. Beg, M.S.; Gupta, A.; Stewart, T.; Rethorst, C.D. Promise of Wearable Physical Activity Monitors in Oncology Practice. J. Oncol. Pract. 2017, 13, 82-89. doi:10.1200/JOP.2016.016857.

5. Kelly, C.M.; Shahrokni, A. Moving beyond Karnofsky and ECOG Performance Status Assessments with New Technologies. J. Oncol. 2016, 2016, 1-13. doi:10.1155/2016/6186543.

6. Ferriolli, E.; Skipworth, R.J.E.; Hendry, P.; Scott, A.; Stensteth, J.; Dahele, M.; Wall, L.; Greig, C.; Fallon, M.; Strasser, F.; et al. Physical Activity Monitoring: A Responsive and Meaningful Patient-Centered Outcome for Surgery, Chemotherapy, or Radiotherapy? J. Pain Symp. Manag. 2012, 43, 1025-1035. doi:10.1016/ j.jpainsymman.2011.06.013.

7. Gresham, G.; Schrack, J.; Gresham, L.M.; Shinde, A.M.; Hendifar, A.E.; Tuli, R.; Rimel, B.J.; Figlin, R.; Meinert, C.L.; et al. Wearable activity monitors in oncology trials: Current use of an emerging technology. Contemp. Clin. Trials 2018, 64, 13-21. doi:10.1016/j.cct.2017.11.002.

8. Hartman, S.J.; Nelson, S.H.; Weiner, L.S. Patterns of Fitbit Use and Activity Levels Throughout a Physical Activity Intervention: Exploratory Analysis from a Randomized Controlled Trial. JMIR mHealth uHealth 2018, 6, e29. doi:10.2196/mhealth.8503.

9. Redman, L.M.; Gilmore, L.A.; Breaux, J.; Thomas, D.M.; Elkind-Hirsch, K.; Stewart, T.; Hsia, D.S.; Burton, J.; Apolzan, J.W.; Cain, L.E.; et al. Effectiveness of SmartMoms, a Novel eHealth Intervention for Management of Gestational Weight Gain: Randomized Controlled Pilot Trial. JMIR mHealth uHealth 2017, 5, e133. doi:10.2196/mhealth.8228.

10. Coughlin, S.S.; Hatzigeorgiou, C.; Anglin, J.; Xie, D.; Besenyi, G.M.; De Leo, G.; Stewart, J.; Wilkins, T. Healthy lifestyle intervention for adult clinic patients with type 2 diabetes mellitus. Diabetes Manag. Lond. Engl. 2017, 7, 197-204. 
11. Dondzila, C.J.; Lewis, C.A.; Lopez, J.R.; Parker, T.M. Congruent Accuracy of Wrist-worn Activity Trackers during Controlled and Free-living Conditions. Int. J. Exerc. Sci. 2018, 11, 575-584.

12. Blarigan, E.L.V.; Kenfield, S.A.; Tantum, L.; Cadmus-Bertram, L.A.; Carroll, P.R.; Chan, J.M. The Fitbit One Physical Activity Tracker in Men With Prostate Cancer: Validation Study. JMIR Cancer 2017, 3, e5. doi:10.2196/cancer.6935.

13. Dominick, G.M.; Winfree, K.N.; Pohlig, R.T.; Papas, M.A. Physical Activity Assessment Between Consumerand Research-Grade Accelerometers: A Comparative Study in Free-Living Conditions. JMIR mHealth uHealth 2016, 4, e110. doi:10.2196/mhealth.6281.

14. Bai, Y.; Welk, G.J.; Nam, Y.H.; Lee, J.A.; Lee, J.M.; Kim, Y.; Meier, N.F.; Dixon, P.M. Comparison of Consumer and Research Monitors under Semistructured Settings. Med. Sci. Sports Exerc. 2016, 48, 151. doi:10.1249/MSS.0000000000000727.

15. Moreno, S.; Damas, M.; Pomares, H.; Moral-Munoz, J.A.; Banos, O. First Approach to Automatic Performance Status Evaluation and Physical Activity Recognition in Cancer Patients. In 2016 15th International Conference on Ubiquitous Computing and Communications and 2016 8th International Symposium on Cyberspace and Security (IUCC-CSS); IEEE: Piscataway, NJ, USA, 2016; pp. 116-123.

16. Nyrop, K.A.; Deal, A.M.; Choi, S.K.; Wagoner, C.W.; Lee, J.T.; Wood, A.; Anders, C.; Carey, L.A.; Dees, E.C.; Jolly, T.A.; et al. Measuring and understanding adherence in a home-based exercise intervention during chemotherapy for early breast cancer. Breast Cancer Res. Treat. 2018, 168, 43-55. doi:10.1007/s10549-017-4565-1.

17. Feehan, L.M.; Geldman, J.; Sayre, E.C.; Park, C.; Ezzat, A.M.; Yoo, J.Y.; Hamilton, C.B.; Li, L.C. Accuracy of Fitbit Devices: Systematic Review and Narrative Syntheses of Quantitative Data. JMIR mHealth uHealth 2018, 6, e10527. doi:10.2196/10527.

18. Tudor-Locke, C.; Bassett, D.R., Jr. How many steps/day are enough? Sports Med. 2004, 34, 1-8.

19. Reid, R.E.R.; Insogna, J.A.; Carver, T.E.; Comptour, A.M.; Bewski, N.A.; Sciortino, C.; Andersen, R.E. Validity and reliability of Fitbit activity monitors compared to ActiGraph GT3X+ with female adults in a free-living environment. J. Sci. Med. Sport 2017, 20, 578-582. doi:10.1016/j.jsams.2016.10.015.

20. Ummels, D.; Beekman, E.; Theunissen, K.; Braun, S.; Beurskens, A.J. Counting Steps in Activities of Daily Living in People With a Chronic Disease Using Nine Commercially Available Fitness Trackers: Cross-Sectional Validity Study. JMIR mHealth uHealth 2018, 6, e70. doi:10.2196/mhealth.8524.

21. Alharbi, M.; Bauman, A.; Neubeck, L.; Gallagher, R. Validation of Fitbit-Flex as a measure of free-living physical activity in a community-based phase III cardiac rehabilitation population. Eur. J. Prev. Cardiol. 2016, 23, 1476-1485. doi:10.1177/2047487316634883.

22. Diaz, K.M.; Krupka, D.J.; Chang, M.J.; Peacock, J.; Ma, Y.; Goldsmith, J.; Schwartz, J.E.; Davidson, K.W.

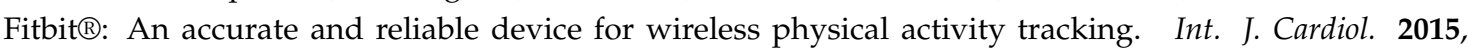
185, 138-140. doi:10.1016/j.ijcard.2015.03.038.

23. Winfree, K.N.; Dominick, G. Modeling Clinically Validated Physical Activity Assessments Using Commodity Hardware. IEEE J. Biomed. Health Inform. 2018, 22, 335-345. doi:10.1109/JBHI.2017.2787461.

24. Thiebaud, R.S.; Funk, M.D.; Patton, J.C.; Massey, B.L.; Shay, T.E.; Schmidt, M.G.; Giovannitti, N. Validity of wrist-worn consumer products to measure heart rate and energy expenditure. Digit. Health 2018, 4, 2055207618770322. doi:10.1177/2055207618770322.

(c) 2018 by the authors. Licensee MDPI, Basel, Switzerland. This article is an open access article distributed under the terms and conditions of the Creative Commons Attribution (CC BY) license (http:/ / creativecommons.org/licenses/by/4.0/). 